in vivo $33: 1713-1720$ (2019)

doi:10.21873/invivo.11661

Review

\title{
Understanding the Role of ztor in Aging-related Diseases Using the Zebrafish Model
}

\author{
ENG-SOON KHOR ${ }^{1}$, SUZITA MOHD NOOR ${ }^{2}$ and POOI-FONG WONG ${ }^{1}$ \\ ${ }^{1}$ Department of Pharmacology, Faculty of Medicine, University of Malaya, Kuala Lumpur, Malaysia; \\ ${ }^{2}$ Department of Biomedical Science, Faculty of Medicine, University of Malaya, Kuala Lumpur, Malaysia
}

\begin{abstract}
The mammalian target of rapamycin (mTOR), a $289 \mathrm{kDa}$ serine/threonine protein kinase of the phosphoinositide 3-kinase (PI3K)-related family is known for its role in regulating lifespan and the aging process in humans and rodents. Aging in zebrafish very much resembles aging in humans. Aged zebrafish often manifest with spinal curvature, cataracts and cognitive frailty, akin to human age-related phenotypical effects such as osteoarthritis, dwindling vision and cognitive dysfunction. However, the role of the zebrafish orthologue of $m T O R$, ztor, is less defined in these areas. This review paper discusses the tale of growing old in the zebrafish, the physiological roles of ztor in normal developmental processes and its involvement in the pathogenesis of aging-related diseases such as metabolic disorders and cancers.
\end{abstract}

Zebrafish is a vertebrate organism that has been in use since the 1970s as a model for studying developmental biology. About a decade later, the first large-scale vertebrate mutagenesis screen led to the discovery of numerous zebrafish mutations homologous to those involved in human diseases (1). Zebrafish is an ideal vertebrate model organism in biomedical research, owing to its extensively-characterized vertebrate biology, and well-conserved molecular and cellular physiology (2). In terms of handling, zebrafish has invertebrate-like technical advantages over the other animal models such as rodents. The zebrafish has many physiological and anatomical characteristics similar to those of the

This article is freely accessible online.

Correspondence to: Pooi-Fong Wong, Department of Pharmacology, Faculty of Medicine, University of Malaya, 50603 Kuala Lumpur, Malaysia. Tel: +603 79677022 (ext. 2065), e-mail: wongpf@um.edu.my

Key Words: Aging-related diseases, developmental, mTOR, zebrafish, ztor, review. vertebrates but with added advantages of possessing high fecundity and rapid embryogenesis which enable large-scale screening akin to that in the invertebrates (3).

Zebrafish is also an ideal model for studying organismal aging. The gradual aging processes that occur in zebrafish resemble those in humans (4). Age-dependent physiological changes in aged zebrafish include muscle abnormalities leading to spinal curvature (5), eye cataracts (6) and deterioration in cognitive functions (7). However, unlike humans, zebrafish have constitutively active telomerase activity. The length of zebrafish telomere $(15-20 \mathrm{~kb})$ is slightly longer than that of humans (10-15 kb). It has been reported that zebrafish telomere length and telomerase expression increase from the embryonic stage to adulthood and then decrease drastically in aged zebrafish (8). Since telomere length and telomerase are involved in aging and cancer susceptibility, analyzing the genetic backgrounds of different developmental profiles in zebrafish could facilitate the study of telomere biology in aging-related diseases (9).

Numerous studies have shown that cellular senescence drives biological aging at the organismal level (10). In this regard, the mammalian target of rapamycin (mTOR) has been extensively linked to both cellular senescence (11-13) and aging $(14,15)$. mTOR activation is stimulated by the availability of nutrients and growth factors via increased phosphorylation of its downstream effectors. These effectors include S6 kinase, ribosomal protein S6 and 4E-BP1 that control protein synthesis by regulating initiation, elongation factors and the biogenesis of ribosomes $(16,17)$. mTOR is best known for its role as a central regulator of cell proliferation, cell growth and metabolism. However, Hall (2016) discovered that mTOR controls cell growth rather than cell proliferation. A study on yeast revealed that TOR mutants behaved differently from the bona fide cell cycle mutants, whereby cdc28 mutant cells continuously grew-up to four times as large as a normal cell despite cell proliferation halting in $\mathrm{G}_{1}$ phase. In contrast, TOR mutant cells did not grow to become as large when cell division was 
arrested in $G_{1}$ phase (18). Hall's study further unveiled defects in protein synthesis in TOR-deficient cells, wherein these cells exhibit altered translation initiation of cell cycle proteins. Therefore, Hall (2016) proposed a paradigm change in the role of TOR; one that directly controls cell growth (e.g. macro-molecular synthesis) but indirectly regulates cell proliferation (18). Aberrant activation of mTOR complex 1 (mTORC1) has been shown to result in cellular and tissue hypertrophy (19) leading to cellular senescence. The phenomenon of hypertrophic cells is the result of prolonged continuous cell growth (increase in cell size or mass) without cell division (20) which is often associated with cellular senescence phenotype.

The zebrafish target of rapamycin (ztor) orthologue has $90 \%$ homology with the mTOR (21) but the role of this highly conserved ztor is less well understood (Figure 1). Previous studies have reported that ztor modulates the developmental process of epithelial morphogenesis in the zebrafish intestine (22) while inhibition of ztor has been shown to exert a long-term cardio-protective effect in adult zebrafish. TOR haploinsufficiency in ztor heterozygous zebrafish diminishes doxorubicin (DOX)-induced cardiomyopathy (23). The findings from these studies have highlighted the importance of ztor signaling in intestinal development and cardiomyopathy, but the precise mechanisms involved need to be further investigated.

\section{Phenotypical Changes in Aged Zebrafish Compared to Human}

Zebrafish is used for aging research due to their favorable gerontological characteristics, whereby aging is a gradual process as in most vertebrates, with obvious decline in growth and waning fecundity with age. The average zebrafish life span is about 3 to 4 years, and zebrafish begin to show signs of aging at approximately 2-years-old (24). Zebrafish adults with heterozygous mutations in both telomeric repeat binding factor 2 (terf2) and zebrafish spinster homolog 1 (spnsl) genes displayed senescenceassociated phenotypes. The onset of aging phenotypes is accelerated in the heterozygous mutant fish and is associated with embryonic neural and muscular degenerative phenotypes including high acridine orange staining in the neural tube, and increased reactive oxygen species (ROS) generation in the neural tube and skeletal muscle, and loss of muscle fibers throughout the myotomes (25).

Spinal curvature. Various degrees of spinal curvature have been reported in aged zebrafish (26) with increased frequency and severity of spinal curvatures seen in 1- to 3year-old zebrafish. Age-related abnormalities including bony outgrowths at the joint margins and fractures within the cortical bone contribute to these spinal curvatures (27).
Gerhard et al. (2002) demonstrated that there is no evidence of bone demineralization and compression deformity, which supports the hypothesis that loss of skeletal muscle due to muscle degeneration is the cause of abnormal spinal curvature (5). These naturally developing age-related changes in aged zebrafish spines bring to mind a range of similar human pathologies associated with degenerative joint disease such as osteoporosis, articular cartilage erosion and osteophytosis in osteoarthritis (28).

Vision degeneration. Increased cataract formation is associated with aging in adult zebrafish. Proteins $\beta \gamma$ - and $\gamma \mathrm{C}$-crystallin in the eye lens are vital for preserving lens transparency. Age-related modifications of crystallin proteins result in light scattering and inferior image projection on the retina. The concomitant loss of lens transparency gradually leads to opacity or cataracts $(29,30)$. Reis et al. (2013) identified a novel $\beta \gamma$-crystallin superfamily gene, $C R Y B A 2$, and found that its p.Val50Met mutation is highly associated with autosomal dominant congenital cataracts. Intriguingly, zebrafish orthologues of $C R Y B A 2$, cryba $2 a$ and cryba $2 b$ are highly expressed during early lens development, suggestive of a role in congenital cataract formation (31). Meanwhile, a zebrafish crygc gainof-function study was performed by microinjecting human mutant CRYGC p.Gly129Cys mRNA into single-cell embryos. Over-expression of the p.Gly129Cys mutant encoding $\gamma \mathrm{C}$-crystallin protein led to the downregulation of lens specific genes in zebrafish including lens intrinsic membrane protein 2.3 ( $\lim 2.3$ ) and connexion $23(\mathrm{cx} 23)$. The result was an increase in lens opacity with concomitant cataract phenotype in zebrafish (32). Correspondingly, the expression of $\alpha$-crystallin protein in the eye lens is markedly increased in aged zebrafish (33).

Cognitive decline. Aging-related cognitive decline is also observed in zebrafish. There is growing evidence of the learning process becoming protracted in old zebrafish. Memory- and avoidance-based tasks are underperformed by aged wild-type zebrafish. Cognitive response to temporal cues is slower in 3-year-old wild-type zebrafish compared to that of younger 1-year-old zebrafish, as indicated by the diminished efficiency of entraining new timing of restricted food administration. In addition, reduced exploratory and increased stereotypic behaviors are observed in aged zebrafish, with alterations in behavioral parameters seen during aging such as a decline in alteration rate prior to the development of stereotypy (34). Likewise, humans suffer from cognitive frailty such as memory loss and slower rate of learning with advancing age (35). Other studies have compared the cognitive abilities of young 1-year-old zebrafish and older 2-year-old zebrafish (36). Young zebrafish possess positive learning abilities with significant 


\begin{tabular}{|c|c|c|c|c|c|c|c|c|c|c|c|c|c|c|c|c|c|}
\hline \multicolumn{12}{|c|}{ Description } & \multirow{2}{*}{$\begin{array}{l}\text { Max } \\
\text { Score } \\
4694 \\
\end{array}$} & \multirow{2}{*}{$\begin{array}{c}\text { Total } \\
\text { Score } \\
4694 \\
\end{array}$} & \multirow{2}{*}{$\begin{array}{c}\text { Query } \\
\text { Cover } \\
99 \%\end{array}$} & \multirow{2}{*}{$\begin{array}{c}\text { E } \\
\text { value } \\
0.0\end{array}$} & \multirow{2}{*}{$\begin{array}{c}\text { Per. } \\
\text { Ident } \\
90.13 \% \\
\end{array}$} & \multirow{2}{*}{$\begin{array}{c}\text { Accession } \\
\text { NP } 001070679.2 \\
\end{array}$} \\
\hline serine/threonir & protein $\mathrm{k}$ & nase mTOR ID & reriol & & & & & & & & & & & & & & \\
\hline Sequence ID & Start & 200 & 400 & 600 & 800 & 1000 & 1200 & 1400 & 1600 & 1800 & 2000 & 2200 & 2400 & \multicolumn{2}{|c|}{2600} & 2884 End & Organism \\
\hline$\frac{\text { NP } 004949.1}{\text { NP } 001070679.2}$ & \begin{tabular}{|l|l|} 
& 16 \\
& 2 \\
\end{tabular} & 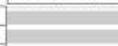 & 1 & & & 1 & & $\frac{1}{1}$ & 11 & 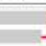 & $\begin{array}{ll}-1 \\
-1\end{array}$ & -41 & \|\|$-1\|\|$ & $\begin{array}{l}4 x-141 \\
4-111\end{array}$ & 4 & 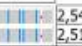 & \begin{tabular}{|l} 
Homo sapiens \\
Danio rerio \\
\end{tabular} \\
\hline
\end{tabular}

Figure 1. Protein sequence alignment of mTOR (Homo sapiens, accession no. NP_004949.1) and its orthologue, ztor (Danio rerio, accession np. NP_001070679.2) performed using BLASTP 2.9.0+, showing 90.13\% homology between the former and latter sequences (upper panel). The lower panel shows the alignment of both sequences viewed using Multiple Sequence Alignment Viewer 1.10.1.

Table I. The involvement of ztor in age-related diseases in zebrafish models.

\begin{tabular}{ll}
\hline Age-related diseases (ARDs) & Roles \\
\hline Obesity & - ztor over-expression enhances adipogenesis \\
Type 2 diabetes & - ztor activation impairs the insulin pathway and inhibits autophagy \\
Cancer & - ztor inhibition reduces tumour sizes and suppresses tumour-associated angiogenesis \\
Age-related retinal neuropathy & - ztor inhibition ameliorates mitochondrial function at the retinal ganglion cell layer \\
Cardiovascular disease & - ztor inhibition reduces angiogenesis \\
& - ztor activation induces vascular malformation \\
& - ztor activation by toxicant causes cardiomyocyte injury
\end{tabular}

improvements in performance seen in the higher number of correct choices made in a color discrimination task compared to the older zebrafish. Moreover, it was shown in a spatial cognition test that young zebrafish needed less search time for a baited hole in the open field maze over the course of the experiment, whereas older zebrafish needed more time in the maze (36). Such changes in the physical performances of aged zebrafish are akin to age-dependent human cognitive impairment during old age $(26,37)$.

Cardiovascular dysfunction. In zebrafish, age-related functional modifications are highly associated with pathophysiological changes in the heart, not unlike the progressive decline of cardiovascular functions leading to increased risk of hypertension, atherosclerosis, and myocardial infarction in humans. Zebrafish heart rate is significantly reduced, with an increase in cardiac arrhythmias or irregular heartbeat in 52-month-old zebrafish compared to 8-month-old zebrafish. There is also an increase of enddiastolic ventricular dimensions, concomitant with a decrease in ventricular compliance and impaired relaxation during aging (38). In addition, aged zebrafish exhibit higher myocardial density of the ventricle and smaller epicardial vessels than that of young zebrafish (26). Consequently, deteriorating cardiac function in aged zebrafish leads to pathological conditions like cardiac hypertrophy and dysfunction comparable to cardiovascular diseases that manifest in humans.

\section{The Role of ztor in Age-related Diseases in the Zebrafish Model}

Although aging and age-related diseases (ARDs) were once thought of as separate entities, recent epidemiological and experimental data show ARDs are parts of a continuum and share similar basic and cellular mechanisms (39). It has been proposed that the aging process, including the onset of ARDs, is driven by the over-activation of signal transduction pathways, in particular, one involving mTOR-driven growth, whereby normal growth and other cellular functions are exacerbated, causing homeostasis deregulation and organ dysfunction and damage $(18,40)$. While the role of mTOR modulation in human aging and in other model organisms are extensively studied (41-43), the role of its highly conserved orthologue ztor is less explored in zebrafish in relation to ARDs. Subsequent sections will discuss ztor involvement in various ARD models in zebrafish (Table I).

The expression of ztor is temporally and spatially dynamic in zebrafish, and is ubiquitous during early embryogenesis which suggests that ztor is pivotal for zebrafish development and growth (44). ztor is detected at 6, 18 and $24 \mathrm{~h}$ postfertilization (hpf) (45) and has been shown to be expressed in the head (44) and developing gut from 35 to $57 \mathrm{hpf}$ (21, 44). ztor involvement in zebrafish development and growth is well described. These include the requirement for ztor activation for gut epithelial morphogenesis (46), axon regeneration (47), synaptic reinforcement and formation of 
new connections for learning and memory (48, 49), angiogenesis (50) and cardiac trabeculation (51). Conversely, over-activation of ztor can lead to an early onset of epilepticlike phenotypic features in zebrafish (52). Since zebrafish exhibit manifestations of aging that are similar to that seen in humans, it is possible to establish models of ARDs such as obesity, type 2 diabetes, cancer, cognitive impairment and age-related retinal neuropathy/oculopathy to facilitate fundamental studies for the understanding of the pathogenesis of these disorders as well as for drug discovery and testing of novel therapeutic strategies.

Obesity. Zebrafish obesity model can be generated by expressing the active form of human AKT1 (myrAKT1) driven by zebrafish skin-specific keratin4 (krt4) promoter in one-cell stage zebrafish embryos. The myrAKT1 is a myristolyation sequence of AKT1 gene (53) that has been modified to bypass the activation of phosphoinositides 3,4,5trisphosphate (PIP3) and PIP2 by PI3K. In humans, myrAKT1 is resistant to inhibition by PTEN, a negative regulator of PI3K pathway. The myrAkt1 transgene zebrafish, $\operatorname{Tg}(k r t 4: H s a . m y r A k t 1) c y 18$ exhibits severely obese phenotypes including hypertrophic and hyperplastic skin growth. Additionally, body weight gain, abnormal fat tissue accumulation and blood glucose intolerance are evident in adult $\operatorname{Tg}($ krt4:Hsa.myrAkt1)cy 18 zebrafish. More importantly, constitutively activated akt signaling upregulates its downstream proteins including ztor and S6 protein kinase (70s6k), and activation of this pathway has been substantially confirmed in obese rat (54). Inversely, ztor suppression in Tg(krt4:Hsa.myrAkt1)cy18 zebrafish at 120 hpf could ameliorate the obese phenotype by markedly decreasing the percentage of hypertrophic skin (55). There is a role for ztor in obesity and its precise functions in adipogenesis, lipid metabolism and thermogenesis such as those reported for mTOR (56) require further investigation.

Type 2 diabetes. The roles of mTOR in glucose and lipid metabolism and diabetes have been extensively studied (57, 58). Type 2 diabetes can be successfully induced in zebrafish by a daily high fat diet consisting of brine shrimp and $1 \%$ egg yolk for 10 weeks. Zebrafish on this long-term high fat diet exhibit clinical signs of obesity such as increased body weight and more lipid vacuoles in liver cells. The insulin signaling pathway is also disrupted with the elevation of fasting blood glucose and insulin mRNA levels in the liver and the downregulation of insulin receptor substrate-2 (irs-2) and glucose transporter 2 (glut2) which mediate the effects of insulin. Interestingly, ztor level is increased $(59,60)$ and is linked to its inhibitory role of autophagy in type 2 diabeteslike zebrafish (61). This is in agreement with previous reports of disruption of autophagy by fatty acid accumulation and modulation by the insulin pathway $(62,63)$. These studies have established the involvement of ztor in type 2 diabetes in zebrafish and could be explored for in-depth studies of ztor in lipid regulation and glucose metabolism.

Cancers. Kim et al. (2013) created a zebrafish cancer model by inducing tsc 2 heterozygous mutation in a p53 mutant background to study cancer pathogenesis. This mutant developed eye tumors, angioma-like tumors near the pectoral fin, melanoma-like tumors on the head and dorsal fin and abdominal tumors (64). It was observed that tumorigenesis is enhanced in tsc2;p53 compound mutant zebrafish compared to p53 mutant zebrafish. In addition, hif1-alpha, hif2-alpha and vegf-c which are important regulators of malignant progression in cancer $(65,66)$ are increased in tsc2;p53 compound mutant zebrafish. This is exemplified by the augmented neo-angiogenesis and vasodilated blood vessels in tumors. ztor signaling was shown to be upregulated in malignant abdominal tumors and eye tumors. Protein levels of downstream and upstream effectors of ztor including s6 kinase and 4ebp1 and akt were upregulated in tsc2;p53 compound mutant zebrafish. In contrast, inhibition of ztor signaling by rapamycin treatment for 2 weeks on the eye tumor, 3 days on the angioma-like tumor at the pectoral fin and 2 days on abdominal tumor, respectively, resulted in shrinking tumor sizes and disrupted tumor-associated angiogenesis (64). Using a zebrafish xenograft model, Chiang et al. (2016) showed that the loss-of-function of PTEN augmented the proliferation of human breast carcinoma (MCF-7) cells. In addition, in vivo metastasis was evidenced by marked dissemination of PTEN-knockdown MCF-7 cells from the primary injection site at the hindbrain ventricle of 48 hpf zebrafish embryos (67). Moreover, epithelialmesenchymal transition (EMT)-inducing transcription factors such as Zeb-1, Zeb-2, Snail, Slug and Twist (68) that are vital for cancer metastasis process were increased in the PTENknockdown MCF-7 cells. Since PTEN is the upstream negative regulator of MTOR in the PI3K/AKT/MTOR pathway, it is be suggested that ztor signaling could be implicated in breast cancer metastasis. These findings highlight a significant role for ztor signaling in tumorigenesis and metastasis of cancer cells (69).

Age-related retinal disease. Abnormal activation of mTOR signaling is reported to lead to retinal pigment epithelial degeneration in humans (70). The role of ztor in retinal disease is unclear but ztor expression is higher in aging retinas of wild-type AB zebrafish. Retinal degeneration in aged zebrafish is linked to a decline in mitochondrial integrity and gene copy number. Aged zebrafish retinas show down-regulation of the pink1 protein, mitohondrial kinase that mediates mitochondrial autophagy (mitophagy). Regulation of mitophagy is essential to maintain mitochondrial function by eliminating damaged mitochondria through selective autophagic process (71). In 
addition, impaired mitochondrial fusion and induced mitochondrial fission are evident in aging zebrafish retinas as exemplified by the reduced $\mathrm{mfn} 2$ and increased fis 1 protein expressions. These defects in mitochondrial quality and fusion/fission reflect the mitochondrial dysfunction that occurs during aging (72). Intriguingly, resveratrol treatment not only improves mitochondrial function but also inhibits elevated ztor activity in aging retinas. Protein expression of ztor is markedly reduced at the retinal ganglion cell layer (GCL) after ten days of resveratrol treatment (73). The question remains on how ztor is linked to mitochondrial dysfunction in age-related retinal neuropathy in older zebrafish.

Cardiovascular disease. ztor plays an important role in zebrafish developmental processes as is clearly illustrated by the defective formation of sub-intestinal vessels (SIVs) and inter-segmental vessels (ISVs) in zebrafish larvae upon inhibition of ztor signaling (74). Inhibition of ztor signaling also hampered endothelial cell proliferation and angiogenesis (50). Ras p21 protein activator 1 (rasa1) and ephrin type-B receptor $\mathrm{B} 4 \mathrm{a}(\mathrm{ephb} 4 \mathrm{a})$ are vital for angiogenesis and deficiency of these two proteins causes ISV defect, arteriovenous malformation and caudal vessel deformity. Correspondingly, pre-treatment with mTOR inhibitor, rapamycin, could reverse abnormal blood vessel phenotypes (75) which suggests that ztor activation causes vascular malformation.

The role of ztor in the development of cardiovascular disease in adult zebrafish has also been reported. Widespread use of the pesticide dieldrin has been shown to render humans highly susceptible to cardiovascular disease $(76,77)$. Recently, cardiac arrest and ventricular fibrillation were induced by dieldrin in 6-month-old AB strain zebrafish. ztor signaling is also constitutively activated with compromised lysosomal physiology in the dieldrin-exposed zebrafish. Upon dieldrin-induced ztor activation, inflammatory genes were up-regulated while lysosomal genes were downregulated, leading to increased inflammatory response and reduced autophagy activity which are the risk factors of zebrafish cardiomyocyte injury (78). This study established that ztor, like its counterpart, mTOR is important in autophagy for the clearance of toxic or dysfunctional protein aggregates.

\section{Conclusion}

This review summarizes the phenotypical changes of aging in zebrafish. The close resemblance of these phenotypical changes to human makes zebrafish a suitable model to study human aging as well as ARDs. mTOR is a master regulator of cellular processes in human. There is now increasing evidence for the role of ztor, its zebrafish orthologue, in normal developmental processes as well as in the pathogenesis and progression of certain ARDs. However, there remain many gaps to be filled when it comes to understanding the role of ztor and ARD pathogenesis in zebrafish disease models that can then be translated and correlated to higher vertebrates, particularly humans. Once the roles of ztor are defined in ARDs, respective zebrafish disease models for highthroughput drug screening and testing can be established for the identification of potentially appealing therapeutic targets. Collectively, this review provides an important reference for leveraging the zebrafish to comprehend the process of aging and its associated disorders.

\section{Conflicts of Interest}

The Authors declare no conflict of interest regarding this study.

\section{Authors' Contributions}

ES Khor performed literature reviews and drafted the manuscript. SM Noor and PF Wong conceived the study design and revised the manuscript. All Authors have read and approved the final manuscript.

\section{Acknowledgements}

The Authors thank the University of Malaya Research Grant (RG500-13HTM) and University of Malaya Postgraduate Research Grant (PG306-2016A) for provision of research funding.

\section{References}

1 Lisu $\mathrm{CH}$, Wen $\mathrm{ZH}$, Lin CS and Chakraborty C: The zebrafish model: Use in studying cellular mechanisms for a spectrum of clinical disease entities. Curr Neurovasc Res 4(2): 111-120, 2007. PMID: 17504209. DOI: 10.2174/156720207780637234

2 Carneiro MC, de Castro IP and Ferreira MG: Telomeres in aging and disease: lessons from zebrafish. Dis Model Mech 9(7): 737748, 2016. PMID: 27482813. DOI: $10.1242 / \mathrm{dmm} .025130$

3 Goldsmith JR and Jobin C: Think Small: Zebrafish as a model system of human pathology. J Biomed Biotechnol, 2012. PMID: 22701308. DOI: $10.1155 / 2012 / 817341$

4 Kishi S: Functional aging and gradual senescence in zebrafish. Ann Ny Acad Sci 1019: 521-526, 2004. PMID: 15247079. DOI: 10.1196/annals.1297.097

5 Gerhard GS, Kauffman EJ, Wang XJ, Stewart R, Moore JL, Kasales CJ, Demidenko E and Cheng KC: Life spans and senescent phenotypes in two strains of Zebrafish (Danio rerio). Exp Gerontol 37(8-9): 1055-1068, 2002. PMID: 12213556. DOI: 10.1016/S0531-5565(02)00088-8

6 Goishi K, Shimizu A, Najarro G, Watanabe S, Rogers R, Zon LI and Klagsbrun M: alpha A-crystallin expression prevents gamma-crystallin insolubility and cataract formation in the zebrafish cloche mutant lens. Development 133(13): 2585-2593, 2006. PMID: 16728471. DOI: 10.1242/dev.02424

7 Adams MM and Kafaligonu H: Zebrafish-A model organism for studying the neurobiological mechanisms underlying cognitive brain aging and use of potential interventions. Front Cell Dev Biol 6, 2018. PMID: 30443547. DOI: 10.3389/Fcell.2018.00135 
8 Anchelin M, Murcia L, Alcaraz-Perez F, Garcia-Navarro EM and Cayuela ML: Behaviour of telomere and telomerase during aging and regeneration in zebrafish. PloS One 6(2): e16955, 2011. PMID: 21347393. DOI: 10.1371/journal.pone.0016955

9 Cayuela ML, Claes KBM, Ferreira MG, Henriques CM, van Eeden F, Varga M, Vierstraete $\mathrm{J}$ and Mione MC: The zebrafish as an emerging model to study DNA damage in aging, cancer and other diseases. Front Cell Dev Biol 6(178), 2019. PMID: 30687705. DOI: $10.3389 /$ Fcell.2018.00178

10 de Magalhaes JP and Passos JF: Stress, cell senescence and organismal ageing. Mech Ageing Dev 170: 2-9, 2018. PMID: 28688962. DOI: $10.1016 /$ j.mad.2017.07.001

11 Walters HE, Deneka-Hannemann S and Cox LS: Reversal of phenotypes of cellular senescence by pan-mTOR inhibition. Aging-Us 8(2): 231-244, 2016. PMID: 26851731. DOI: 10.18632/Aging. 100872

12 Khor ES and Wong PF: Endothelial replicative senescence delayed by the inhibition of MTORC1 signaling involves MicroRNA-107. Int J Biochem Cell B 101: 64-73, 2018. PMID: 29857052. DOI: 10.1016/j.biocel.2018.05.016

13 Wang R, Yu Z, Sunchu B, Shoaf J, Dang I, Zhao S, Caples K, Bradley L, Beaver LM, Ho E, Lohr CV and Perez VI: Rapamycin inhibits the secretory phenotype of senescent cells by a Nrf2-independent mechanism. Aging Cell 16(3): 564-574, 2017. PMID: 28371119. DOI: $10.1111 /$ acel.12587

14 Khor ES, Noor SM and Wong PF: Expression of zTORassociated microRNAs in zebrafish embryo treated with rapamycin. Life Sci 150: 67-75, 2016. PMID: 26916825. DOI: 10.1016/j.lfs.2016.02.076

15 Kolosova NG, Vitovtov AO, Muraleva NA, Akulov AE, Stefanova NA and Blagosklonny MV: Rapamycin suppresses brain aging in senescence-accelerated OXYS rats. Aging-Us 5(6): 474-484, 2013. PMID: 23817674. DOI: 10.18632/ Aging.100573

16 Wang XM and Proud CG: mTORC1 signaling: what we still don't know. J Mol Cell Biol 3(4): 206-220, 2011. PMID: 21138990. DOI: $10.1093 / \mathrm{jmcb} / \mathrm{mjq} 038$

17 Wang XM and Proud CG: The mTOR pathway in the control of protein synthesis. Physiology 21: 362-369, 2006. PMID: 16990457. DOI: 10.1152 /physiol.00024.2006

18 Hall MN: TOR and paradigm change: cell growth is controlled. Mol Biol Cell 27(18): 2804-2806, 2016. PMID: 27634743. DOI: 10.1091/mbc.E15-05-0311

19 Lee $\mathrm{CH}$, Inoki K and Guan KL: mTOR pathway as a target in tissue hypertrophy. Annu Rev Pharmacol 47: 443-467, 2007. PMID: 16968213. DOI: 10.1146/annurev.pharmtox.47.120505. 105359

20 Blagosklonny MV: Cell senescence: Hypertrophic arrest beyond the restriction point. J Cell Physiol 209(3): 592-597, 2006. PMID: 17001692 . DOI: $10.1002 /$ jcp.20750

21 Makky K and Mayer AN: Zebrafish offers new perspective on developmental role of TOR signaling. Organogenesis 3(2): 6769, 2007. PMID: 19279702. DOI: 10.4161/org.3.2.5378

22 Makky K, Tekiela J and Mayer AN: Target of rapamycin (TOR) signaling controls epithelial morphogenesis in the vertebrate intestine. Dev Biol 303(2): 501-513, 2007. PMID: 17222402. DOI: $10.1016 /$ j.ydbio.2006.11.030

23 Ding Y, Sun X, Huang W, Hoage T, Redfield M, Kushwaha S, Sivasubbu S, Lin X, Ekker S and Xu X: Haploinsufficiency of target of rapamycin attenuates cardiomyopathies in adult zebrafish. Circ Res 109(6): 658-669, 2011. PMID: 21757652. DOI: $10.1161 /$ CIRCRESAHA.111.248260

24 Gerhard GS and Cheng KC: A call to fins! Zebrafish as a gerontological model. Aging Cell 1(2): 104-111, 2002. PMID: 12882339. DOI: 10.1046/j.1474-9728.2002.00012.x

25 Kishi S, Bayliss PE, Uchiyama J, Koshimizu E, Qi J, Nanjappa $\mathrm{P}$, Imamura S, Islam A, Neuberg D, Amsterdam A and Roberts TM: The identification of zebrafish mutants showing alterations in senescence-associated biomarkers. Plos Genet 4(8), 2008. PMID: 18704191. DOI: 10.1371/journal.pgen.1000152

26 Sun YY, Fang YH, Xu XL, Lu GP and Chen ZY: Evidence of an association between age-related functional modifications and pathophysiological changes in zebrafish heart. Gerontology 61(5): 435-447, 2015. PMID: 25531915. DOI: 10.1159/0003 69094

27 Hayes AJ, Reynolds S, Nowell MA, Meakin LB, Habicher J, Ledin J, Bashford A, Caterson B and Hammond CL: Spinal deformity in aged zebrafish is accompanied by degenerative changes to their vertebrae that resemble osteoarthritis. Plos One 8(9): e75787, 2013. PMID: 24086633. DOI: 10.1371/journal. pone. 0075787

28 Bendele AM: Animal models of osteoarthritis in an era of molecular biology. J Musculoskelet Neuronal Interact 2(6): 501503, 2002. PMID: 15758375.

29 Jonasova K and Kozmik Z: Eye evolution: Lens and cornea as an upgrade of animal visual system. Semin Cell Dev Biol 19(2): 71-81, 2008. PMID: 18035562. DOI: 10.1016/j.semcdb. 2007.10.005

30 Sharma KK and Santhoshkumar P: Lens aging: Effects of crystallins. Biochim Biophys Acta 1790(10): 1095-1108, 2009. PMID: 19463898. DOI: 10.1016/j.bbagen.2009.05.008

31 Reis LM, Tyler RC, Muheisen S, Raggio V, Salviati L, Han DP, Costakos D, Yonath H, Hall S, Power P and Semina EV: Whole exome sequencing in dominant cataract identifies a new causative factor, CRYBA2, and a variety of novel alleles in known genes. Hum Genet 132(7): 761-770, 2013. PMID: 23508780. DOI: $10.1007 / \mathrm{s} 00439-013-1289-0$

32 Li XQ, Cai HC, Zhou SY, Yang JH, Xi YB, Gao XB, Zhao WJ, Li P, Zhao GY, Tong Y, Bao FC, Ma Y, Wang S, Yan YB, Lu CL and $\mathrm{Ma} \mathrm{X}$ : A novel mutation impairing the tertiary structure and stability of gammaC-crystallin (CRYGC) leads to cataract formation in humans and zebrafish lens. Hum Mut 33(2): 391401, 2012. PMID: 22052681. DOI: 10.1002/humu.21648

33 Greiling TM, Houck SA and Clark JI: The zebrafish lens proteome during development and aging. Mol Vis 15: 23132325, 2009. PMID: 2779061.

34 Yu LL, Tucci V, Kishi S and Zhdanova IV: Cognitive Aging in Zebrafish. Plos One 1(1), 2006. PMID: 19936306. DOI: 10.1371/journal.pone.0000014

35 Bishop NA, Lu T and Yankner BA: Neural mechanisms of ageing and cognitive decline. Nature 464(7288): 529-535, 2010. PMID: 20336135. DOI: 10.1038/nature08983

36 Ruhl T, Jonas A, Seidel NI, Prinz N, Albayram O, Bilkei-Gorzo A and von der Emde G: Oxidation and Cognitive Impairment in the Aging Zebrafish. Gerontology 62(1): 47-57, 2015. PMID: 26183067. DOI: $10.1159 / 000433534$

37 Gilbert MJH, Zerulla TC and Tierney KB: Zebrafish (Danio rerio) as a model for the study of aging and exercise: Physical ability and trainability decrease with age. Exp Gerontol 50: 106113, 2014. PMID: 24316042. DOI: 10.1016/j.exger.2013.11.013 
38 Arbab-Zadeh A, Dijk E, Prasad A, Fu Q, Torres P, Zhang R Thomas JD, Palmer D and Levine BD: Effect of aging and physical activity on left ventricular compliance. Circulation 110(13): 1799-1805, 2004. PMID: 15364801. DOI: 10.1161/ 01.Cir.0000142863.71285.74

39 Franceschi C, Garagnani P, Morsiani C, Conte M, Santoro A, Grignolio A, Monti D, Capri M and Salvioli S: The continuum of aging and age-related diseases: common mechanisms but different rates. Front Med (Lausanne) 5(61), 2018. PMID: 29662881. DOI: $10.3389 /$ fmed.2018.00061

40 Blagosklonny MV: Aging is not programmed Genetic pseudoprogram is a shadow of developmental growth. Cell Cycle 12(24): 3736-3742, 2013. PMID: 24240128. DOI: 10.4161/cc.27188

41 Hertweck M, Gobel C and Baumeister R: C. elegans SGK-1 is the critical component in the Akt/PKB kinase complex to control stress response and life span. Dev Cell 6(4): 577-588, 2004. PMID: 15068796. DOI: 10.1016/S1534-5807(04)00095-4

42 Sharp ZD and Bartke A: Evidence for down-regulation of phosphoinositide 3-kinase/Akt/mammalian target of rapamycin (PI3K/Akt/mTOR)-dependent translation regulatory signaling pathways in Ames dwarf mice. J Gerontol A Biol Sci Med Sci 60(3): 293-300, 2005. PMID: 15860463. DOI: 10.1093/ gerona/60.3.23

43 Harries LW, Fellows AD, Pilling LC, Hernandez D, Singleton A, Bandinelli S, Guralnik J, Powell J, Ferrucci L and Melzer D: Advancing age is associated with gene expression changes resembling mTOR inhibition: Evidence from two human populations. Mech Ageing Dev 133(8): 556-562, 2012. PMID: 22813852. DOI: $10.1016 /$ j.mad.2012.07.003

44 Fleming A and Rubinsztein DC: Zebrafish as a model to understand autophagy and its role in neurological disease. Biochim Biophys Acta 1812(4): 520-526, 2011. PMID: 21256213. DOI: $10.1016 /$ j.bbadis.2011.01.004

45 Sasore $\mathrm{T}$ and Kennedy B: Deciphering combinations of $\mathrm{PI} 3 \mathrm{~K} / \mathrm{AKT} / \mathrm{mTOR}$ pathway drugs augmenting anti-angiogenic efficacy in vivo. Plos One 9(8), 2014. PMID: 25144531. DOI: 10.1371/journal.pone. 0105280

46 Makky K, Tekiela J and Mayer AN: Target of rapamycin (TOR) signaling controls epithelial morphogenesis in the vertebrate intestine. Dev Biol 303(2): 501-513, 2007. PMID: 17222402. DOI: $10.1016 /$ j.ydbio.2006.11.030

47 Diekmann H, Kalbhen P and Fischer D: Active mechanistic target of rapamycin plays an ancillary rather than essential role in zebrafish CNS axon regeneration. Front Cell Neurosci 9, 2015. PMID: 26217179. DOI: 10.3389/Fncel.2015.00251

48 Garelick MG and Kennedy BK: TOR on the brain. Exp Gerontol 46(2-3): 155-163, 2011. PMID: 20849946. DOI: 10.1016/ j.exger.2010.08.030

49 Izquierdo I and Medina JH: Memory formation: The sequence of biochemical events in the hippocampus and its connection to activity in other brain structures. Neurobiol Learn Mem 68(3): 285-316, 1997. PMID: 9398590. DOI: 10.1006/nlme.1997.3799

50 Xue Q, Nagy JA, Manseau EJ, Phung TL, Dvorak HF and Benjamin LE: Rapamycin inhibition of the Akt/mTOR pathway blocks select stages of VEGF-A164-driven angiogenesis, in part by blocking S6Kinase. Arterioscler Thromb Vasc Biol 29(8): 1172-1178, 2009. PMID: 19443844. DOI: 10.1161/ATVBAHA 109.185918

51 Fleming ND, Samsa LA, Hassel D, Qian L and Liu J: Rapamycin attenuates pathological hypertrophy caused by an absence of trabecular formation. Sci Rep 8(1): 8584, 2018. PMID: 29872120. DOI: 10.1038/s41598-018-26843-1

52 de Calbiac H, Dabacan A, Marsan E, Tostivint H, Devienne G, Ishida S, Leguern E, Baulac S, Muresan RC, Kabashi E and Ciura S: Depdc5 knockdown causes mTOR-dependent motor hyperactivity in zebrafish. Ann Clin Translat Neurol 5(5): 510523, 2018. PMID: 29761115. DOI: 10.1002/acn3.542

53 O'Donnell RK, Goldstein WE, Perruzzi C, Benjamin LE and Aird WC: Overexpression of MyrAkt1 in endothelial cells leads to erythropoietin- and BMP4-independent splenic erythropoiesis in mice. PloS One 8(1): e55095, 2013. PMID: 23383068. DOI: 10.1371/journal.pone.0055095

54 Khamzina L, Veilleux A, Bergeron S and Marette A: Increased activation of the mammalian target of rapamycin pathway in liver and skeletal muscle of obese rats: possible involvement in obesity-linked insulin resistance. Endocrinology 146(3): 14731481, 2005. PMID: 15604215. DOI: 10.1210/en.2004-0921

55 Chu CY, Chen CF, Rajendran RS, Shen CN, Chen TH, Yen CC, Chuang CK, Lin DS and Hsiao CD: Overexpression of Akt1 enhances adipogenesis and leads to lipoma formation in zebrafish. PLoS One 7(5): e36474, 2012. PMID: 22623957. DOI: 10.1371 journal.pone. 0036474

56 Cai H, Dong LQ and Liu F: Recent advances in adipose mTOR Signaling and Function: Therapeutic prospects. Trends Pharmacol Sci 37(4): 303-317, 2016. PMID: 26700098. DOI: 10.1016/j.tips.2015.11.011

57 Mao Z and Zhang W: Role of mTOR in glucose and lipid metabolism. Int J Mol Sci 19(7), 2018. PMID: 30011848. DOI: 10.3390/ijms 19072043

58 Zoncu R, Efeyan A and Sabatini DM: mTOR: from growth signal integration to cancer, diabetes and ageing. Nat Rev Mol Cell Biol 12(1): 21-35, 2011. PMID: 21157483. DOI: 10.1038/nrm3025

59 Shimomura I, Matsuda M, Hammer RE, Bashmakov Y, Brown MS and Goldstein JL: Decreased IRS-2 and increased SREBP1c lead to mixed insulin resistance and sensitivity in livers of lipodystrophic and ob/ob mice. Mol Cell 6(1): 77-86, 2000. PMID: 10949029. DOI: Doi 10.1016/S1097-2765(00)00009-5

60 Leturque A, Brot-Laroche E and Le Gall M: GLUT2 mutations, translocation, and receptor function in diet sugar managing. Am J Physiol-Endoc M 296(5): 985-992, 2009. PMID: 19223655. DOI: 10.1152/ajpendo.00004.2009

61 Meng XH, Chen B and Zhang JP: Intracellular insulin and impaired autophagy in a zebrafish model and a cell model of type 2 diabetes. Int J Biol Sci 13(8): 985-995, 2017. PMID: 28924380. DOI: $10.7150 /$ ijbs. 19249

62 Papackova Z, Dankova H, Palenickova E, Kazdova L and Cahova M: Effect of short- and long-term high-fat feeding on autophagy flux and lysosomal activity in rat liver. Physiol Res 61: 67-76, 2012. PMID: 23130905.

63 Mizushima N, Levine B, Cuervo AM and Klionsky DJ: Autophagy fights disease through cellular self-digestion. Nature 451(7182): 1069-1075, 2008. PMID: 18305538. DOI: 10.1038/ nature 06639

64 Kim SH, Kowalski ML, Carson RP, Bridges LR and Ess KC: Heterozygous inactivation of tsc2 enhances tumorigenesis in $\mathrm{p} 53$ mutant zebrafish. Dis Model Mech 6(4): 925-933, 2013. PMID: 23580196. DOI: 10.1242/dmm.011494

65 Vaupel P: The role of hypoxia-induced factors in tumor progression. Oncologist 9: 10-17, 2004. PMID: 15591418. DOI: 10.1634/theoncologist.9-90005-10 
66 Yamamoto Y, Ibusuki M, Okumura Y, Kawasoe T, Kai K, Iyama $\mathrm{K}$ and Iwase $\mathrm{H}$ : Hypoxia-inducible factor 1 alpha is closely linked to an aggressive phenotype in breast cancer. Breast Cancer Res Tr 110(3): 465-475, 2008. PMID: 17805961. DOI: 10.1007/s10549-007-9742-1

67 Chiang KC, Hsu SY, Lin SJ, Yeh CN, Pang JH, Wang SY, Hsu JT, Yeh TS, Chen LW, Kuo SF, Cheng YC, Juang HH: PTEN insufficiency increases breast cancer cell metastasis in vitro and in vivo in a xenograft zebrafish model. Anticancer Res 36(8): 3997-4005, 2016. PMID: 27466505

68 Peinado H, Olmeda D, Cano A: Snail, Zeb and bHLH factors in tumour progression: an alliance against the epithelial phenotype? Nat Rev Cancer 7(6): 415-428, 2007. PMID: 17508028. DOI: $10.1038 / \mathrm{nrc} 2131$

69 Cao L, Li W, Kim S, Brodie SG and Deng CX: Senescence, aging, and malignant transformation mediated by p53 in mice lacking the Brcal full-length isoform. Genes Dev 17(2): 201213, 2003. PMID: 12533509 . DOI: $10.1101 / \mathrm{gad} .1050003$

70 Huang J, Gu S, Chen M, Zhang SJ, Jiang Z, Chen X, Jiang C, Liu G, Radu RA, Sun X, Vollrath D, Du J, Yan B and Zhao C: Abnormal mTORC1 signaling leads to retinal pigment epithelium degeneration. Theranostics 9(4): 1170-1180, 2019. PMID: 30867823. DOI: 10.7150/thno.26281

71 Palikaras K, Lionaki E and Tavernarakis N: Coordination of mitophagy and mitochondrial biogenesis during ageing in $\mathrm{C}$. elegans. Nature 521(7553): 525-528, 2015. PMID: 25896323. DOI: $10.1038 /$ nature 14300

72 Gonzalez-Freire M, de Cabo R, Bernier M, Sollott SJ, Fabbri E, Navas $\mathrm{P}$ and Ferrucci L: Reconsidering the Role of Mitochondria in Aging. The journals of gerontology Series A, Biological sciences and medical sciences 70(11): 1334-1342, 2015. PMID: 25995290. DOI: $10.1093 /$ gerona/glv070

73 Wang N, Luo Z, Jin M, Sheng W, Wang HT, Long X, Wu Y, Hu $\mathrm{P}, \mathrm{Xu} \mathrm{H}$ and Zhang $\mathrm{X}$ : Exploration of age-related mitochondrial dysfunction and the anti-aging effects of resveratrol in zebrafish retina. Aging (Albany NY), 2019. PMID: 31105084. DOI: 10.18632/aging.101966
74 Khor ES, Noor SM and Wong PF: Expression of zTORassociated microRNAs in zebrafish embryo treated with rapamycin. Life Sci 150: 67-75, 2016. PMID: 26916825. DOI: $10.1016 /$ j.lfs.2016.02.076

75 Kawasaki J, Aegerter S, Fevurly RD, Mammoto A, Mammoto T, Sahin M, Mably JD, Fishman SJ and Chan J: RASA1 functions in EPHB4 signaling pathway to suppress endothelial mTORC1 activity. J Clin Invest 124(6): 2774-2784, 2014. PMID: 24837431. DOI: 10.1172/JCI67084

76 Kim SA, Kim KS, Lee YM, Jacobs DR and Lee DH: Associations of organochlorine pesticides and polychlorinated biphenyls with total, cardiovascular, and cancer mortality in elders with differing fat mass. Environ Res 138: 1-7, 2015. PMID: 25682252. DOI: 10.1016/j.envres.2015.01.021

77 Ljunggren SA, Helmfrid I, Salihovic S, van Bavel B, Wingren G, Lindahl $M$ and Karlsson H: Persistent organic pollutants distribution in lipoprotein fractions in relation to cardiovascular disease and cancer. Environ Int 65: 93-99, 2014. PMID: 24472825. DOI: 10.1016/j.envint.2013.12.017

78 Slade L, Cowie A, Martyniuk CJ, Kienesberger PC and Pulinilkunnil T: Dieldrin augments mTOR signaling and regulates genes associated with cardiovascular disease in the adult zebrafish heart (Danio rerio). J Pharmacol Exp Ther 361(3): 375-385, 2017. PMID: 28385952. DOI: 10.1124/ jpet.116.239806
Received August 6, 2019

Revised August 15, 2019 Accepted August 16, 2019 